\title{
BMJ Open Quality Improving stroke care in Nova Scotia, Canada: a population-based project spanning 14 years
}

\author{
Stephen James Phillips (D) , ${ }^{1}$ Allison Stevens, ${ }^{2}$ Huiling Cao, ${ }^{2}$ Wendy Simpkin, ${ }^{2}$ \\ Jennifer Payne, ${ }^{3}$ Neala Gill ${ }^{2}$
}

To cite: Phillips SJ, Stevens A, Cao $\mathrm{H}$, et al. Improving stroke care in Nova Scotia, Canada: a populationbased project spanning 14 years. BMJ Open Quality 2021;10:e001368. doi:10.1136/ bmjoq-2021-001368

Received 26 January 2021 Accepted 4 September 2021

Check for updates

(C) Author(s) (or their employer(s)) 2021. Re-use permitted under CC BY-NC. No commercial re-use. See rights and permissions. Published by BMJ.

${ }^{1}$ Medicine, Queen Elizabeth II Health Sciences Centre, Halifax, Nova Scotia, Canada

${ }^{2}$ Cardiovascular Health Nova Scotia, Queen Elizabeth II Health Sciences Centre, Halifax, Nova Scotia, Canada

${ }^{3}$ Diagnostic Radiology, Dalhousie University, Halifax, Nova Scotia, Canada

Correspondence to Dr Stephen James Phillips; stephen.phillips@nshealth.ca

\section{ABSTRACT}

Stroke is a complex disorder that challenges healthcare systems. An audit of in-hospital stroke care in the province of Nova Scotia, Canada, in 2004-2005 indicated that many aspects of care delivery fell short of national best practice recommendations. Stroke care in Nova Scotia was reorganised using a combination of interventions to facilitate systems change and quality improvement. The focus was mainly on implementing evidence-based stroke unit care, augmenting thrombolytic therapy and enhancing dysphagia assessment. Key were the development of a provincial network to facilitate ongoing collaboration and structured information exchange, the creation of the stroke coordinator and stroke physician champion roles, and the implementation of a registry to capture information about adults hospitalised because of stroke or transient ischaemic attack. To evaluate the interventions, a longitudinal analysis compared the audit results with registry data for 2012, 2015 and 2019. The proportion of patients receiving multidisciplinary stroke unit care rose from $22.4 \%$ in 2005 to $74.0 \%$ in 2019 . The proportion of patients who received alteplase increased steadily from $3.2 \%$ to $18.5 \%$, and the median delay between hospital arrival and alteplase administration decreased from $102 \mathrm{~min}$ to $56 \mathrm{~min}$, without an increase in intracranial haemorrhage. Dysphagia screening increased from $41.4 \%$ to $77.4 \%$. More patients were transferred from acute care to a dedicated in-patient rehabilitation unit, and fewer were discharged to residential or long-term care. These enhancements did not prolong length-of-stay in acute care. The network was a critical success factor; competing priorities in the healthcare system were the main challenge to implementing change. A multidimensional, multiyear, improvement intervention yielded substantial and sustained improvements in the process and structure of stroke care in Nova Scotia.

"If you want to go fast, go alone. If you want to go far, go together." African proverb

\section{CONTEXT}

Canada is a federation of 10 provinces and 3 territories with a universal healthcare system, where all Canadian citizens and permanent residents have state-insured access to hospital and physician care. The provincial and territorial governments are responsible for the management, organisation and delivery of healthcare services for their residents. Nova Scotia is a province of $55280 \mathrm{~km}^{2}$ (almost two-thirds the size of Scotland, after which it is named) and almost one million people on the east coast of Canada. Geographically the second smallest province in the country, it has the second highest population density with about $40 \%$ living in the urban Halifax region, and $60 \%$ in rural areas.

The Queen Elizabeth II Health Sciences Centre (QEII) in Halifax provides care for about 400000 local residents, tertiary care for all of Nova Scotia and quaternary care for individuals from 3 neighbouring Atlantic provinces. Before the interventions described here, patients who had a stroke in Nova Scotia were managed in 33 hospitals (including the QEII) of varying sizes distributed across 9 separate district health authorities (DHAs) that later became subsumed under the Nova Scotia Health Authority (NSHA).

\section{PROBLEM}

The impetus to improve stroke care in Nova Scotia stemmed from advances in stroke treatment. ${ }^{12}$ Recognising the need for systems change, re-organisation strategies were promoted by the Heart and Stroke Foundation (www.heartandstroke.ca) ${ }^{3}$ and supported by the Networks of Centres of Excellence programme of the Federal Government of Canada (www.nce-rce.gc.ca) through funding of the Canadian Stroke Network.

In 2006, in response to a report submitted by the Heart and Stroke Foundation of Nova Scotia (coauthored by SJP), the government of Nova Scotia announced dedicated funding to enhance stroke care services. To enable this, the government's department of health expanded its provincial programme, Cardiovascular Health Nova Scotia (CVHNS), to include stroke in its mandate of leading province-wide healthcare improvement, 
monitoring and surveillance of cardiovascular disease and stroke care.

This report summarises the impact of efforts led by CVHNS to improve stroke care in Nova Scotia over a 14-year period by promoting systems change and implementing components of the Canadian Stroke Best Practice Recommendations (CSBPR). ${ }^{45}$ At the outset, targets for improvement were not clearly defined. Subsequently, benchmarks were established ${ }^{5}$ and for some performance measures, the targets became more stringent. Here, we present a longitudinal analysis evaluating quantitative changes in the care for adults hospitalised because of stroke or transient ischaemic attack (TIA), complemented by qualitative information from structured stakeholder feedback.

\section{Background}

Stroke treatment came of age in the 1990s when clinical trials demonstrated the efficacy of stroke unit care ${ }^{1}$ and thrombolytic therapy. ${ }^{2}$ Confronting persisting nihilistic attitudes ${ }^{6}$ towards stroke, it was realised that a systems approach ${ }^{7}$ was needed to transform healthcare systems that were unaccustomed to managing stroke in a coordinated manner. Since then, the evidence for stroke unit care has strengthened, ${ }^{8}$ the components of effective stroke unit care have become better defined, ${ }^{59}$ methods for increasing thrombolytic therapy have been explored ${ }^{10}$ and several countries-including Canada $^{4}{ }^{5}$-have produced guidelines for stroke prevention, treatment, rehabilitation and community reintegration. However, large-scale implementation of these guidelines has proved challenging ${ }^{11}$ and remains a work-in-progress in many countries. ${ }^{12}$

\section{Measurement}

Stroke care before re-organisation was evaluated by an audit in which nurses were trained to extract information from the medical records of patients who were hospitalised because of community-onset stroke or TIA during fiscal 2004-2005 using the Registry of the Canadian Stroke Network. ${ }^{13}$ Patients were identified from the Canadian Institute for Health Information (CIHI) Discharge Abstract Database (DAD) ${ }^{14}$ on the basis of a most responsible diagnosis defined by the following International Classification of Diseases-10 codes: I61, I63, I64 and G45 (excluding G45.4).

The impact of the changes led by CVHNS was monitored by continuous data collection that was initiated province wide in July 2011. The data were collected by stroke coordinators in each District Stroke Programme (DSP) using the CVHNS Stroke Registry, which comprised key performance measures derivedby a consensus process involving stakeholders and a working group of CVHNS staff-from the performance measures prescribed in the CSBPR. ${ }^{5}$ To ensure complete case ascertainment, the stroke coordinators identified patients prospectively, and additional cases were detected through quarterly review of the DAD.
The CVHNS Stroke Registry data were used to generate annual reports for decision-makers in the NSHA; custom reports were also available by request from any stakeholder, and stroke coordinators could run a local report at any point in time.

The audit results and data from the CVHNS Stroke Registry for calendar 2012 (the first full year of data collection), 2015 (10 years after the audit) and 2019 (the most recent available data) are shown in table 1 and figure 1. All data were collected without consent because the information was required for quality improvement purposes.

Hospital admission rates were calculated using population denominators obtained from Statistics Canada. Post-thrombolysis intracranial haemorrhage was defined as neurological deterioration and CT scan evidence of any intracranial haemorrhage at any time during hospitalisation, or death within 24 hours of alteplase (ie, typically before any routine follow-up CT scan). Functional status at discharge was measured using the modified Rankin Scale, ${ }^{15}$ with independence defined as a score of $0-2$.

Qualitative data on key success factors for stroke care reorganisation and improvement were collected from various stakeholders between 2010 and 2013 by the stroke coordinators using structured questionnaires that included several open-ended questions. As well, informal feedback flowed as part of the routine communication between CVHNS and the stroke coordinators.

\section{Design}

The overarching aim of the intervention was to promote evidence-informed practice based on the CSBPR. ${ }^{45}$ This required provincial systems change as well as quality improvement efforts. Implementation focused mainly on facilitating coordinated, evidence-based, multidisciplinary stroke unit care, timely treatment of acute ischaemic stroke with intravenous alteplase and improved dysphagia assessment (figure 2). Several other aspects of stroke care (including palliative care, rehabilitation and fast-track outpatient management of TIA) also received attention (see figure 3 ) but are not included in this report.

CVHNS (programme manager (NG), and one staff member) worked at 'arm's length' with the DHAs to implement changes. A network of seven DSPs was created within the NSHA, each with a stroke coordinator and stroke physician champion. The provincial network of DSPs was overseen by CVHNS, but day-to-day operations were the responsibility of the DHAs. The stroke coordinators communicated freely with CVHNS; as well, there were scheduled formal bimonthly group teleconferences, and in-person meetings once or twice times per year. Medical (neurological) input was provided, as needed and through regular meetings, by SJP in his role as clinical advisor to CVHNS. The QEII's stroke team ${ }^{16}$ was also a source of support and guidance. 
Table 1 Summary of demographic data, process, outcome and balancing measures from 2005 (preintervention) to 2019

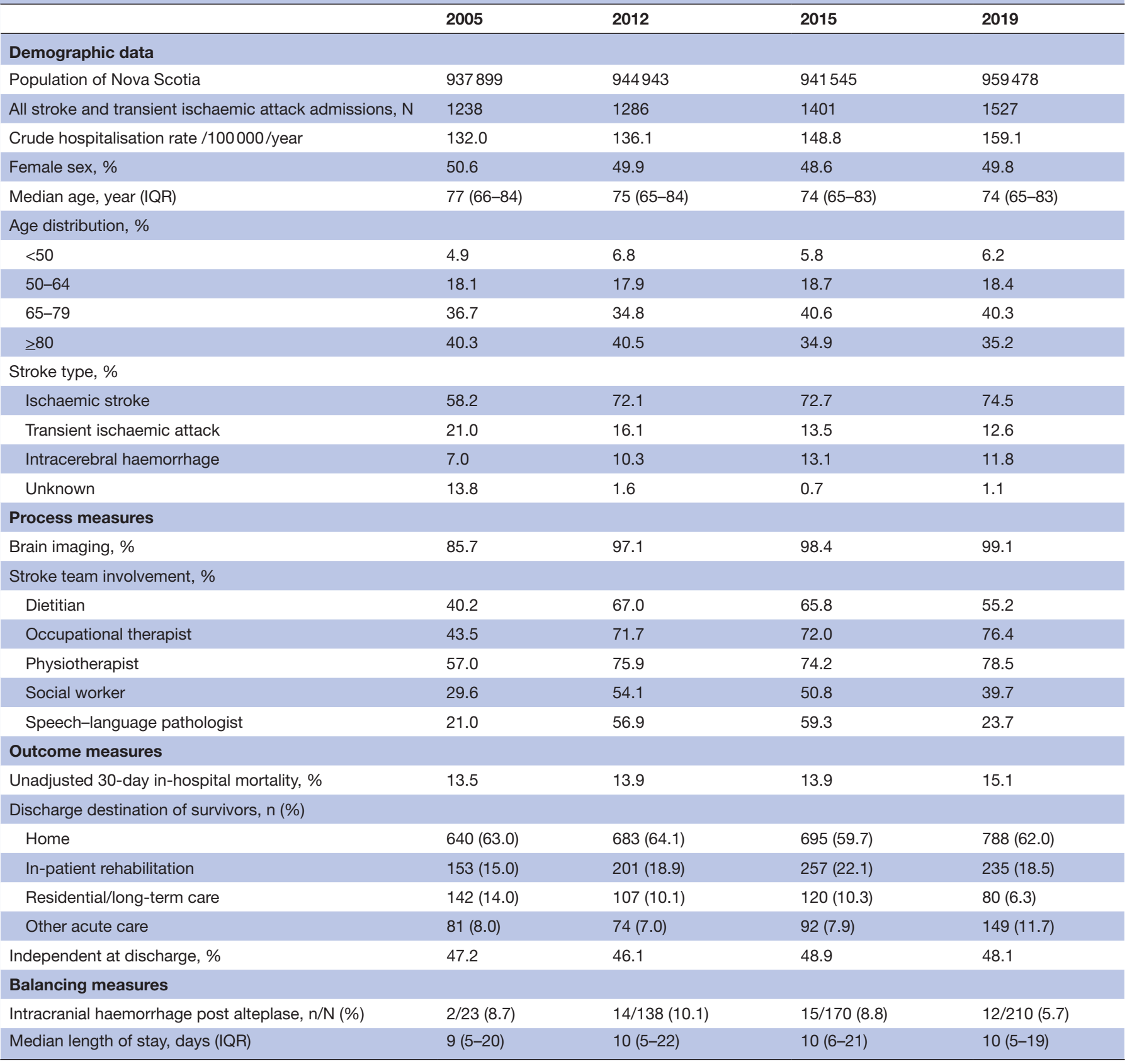

The stroke coordinator was responsible for leading the planning, development, implementation, evaluation and ongoing maintenance of a coordinated, integrated, local DSP. The stroke physician champion role involved participation in and support for systems change, and the provision of education in best practice care. Outside the QEII, the physicians fulfilling this role were family physicians or internal medicine specialists. In most DHAs, a stipend was provided to support the stroke physician champion role to compensate the physician for organisational and administrative work not recompensed through the routine clinical fee-forservice remuneration system. At the QEII, neurologists and specialists in physical medicine and rehabilitation are mainly salaried through an academic funding plan.

\section{Strategy}

The work of enhancing stroke care province wide began in 2007 after the completion of a demonstration project in one of the more rural and remote DHAs. CVHNS defined the minimal case volume required to maintain high quality care as 115 patients/year (based on an average hospital length of stay of 19 days on a six-bed unit, which was the suggested minimum size required to maintain high quality care $^{9}$ ). This definition indicated a need to consolidate stroke care in seven stroke units, ranging in size from 4 to 30 beds, recognising that 


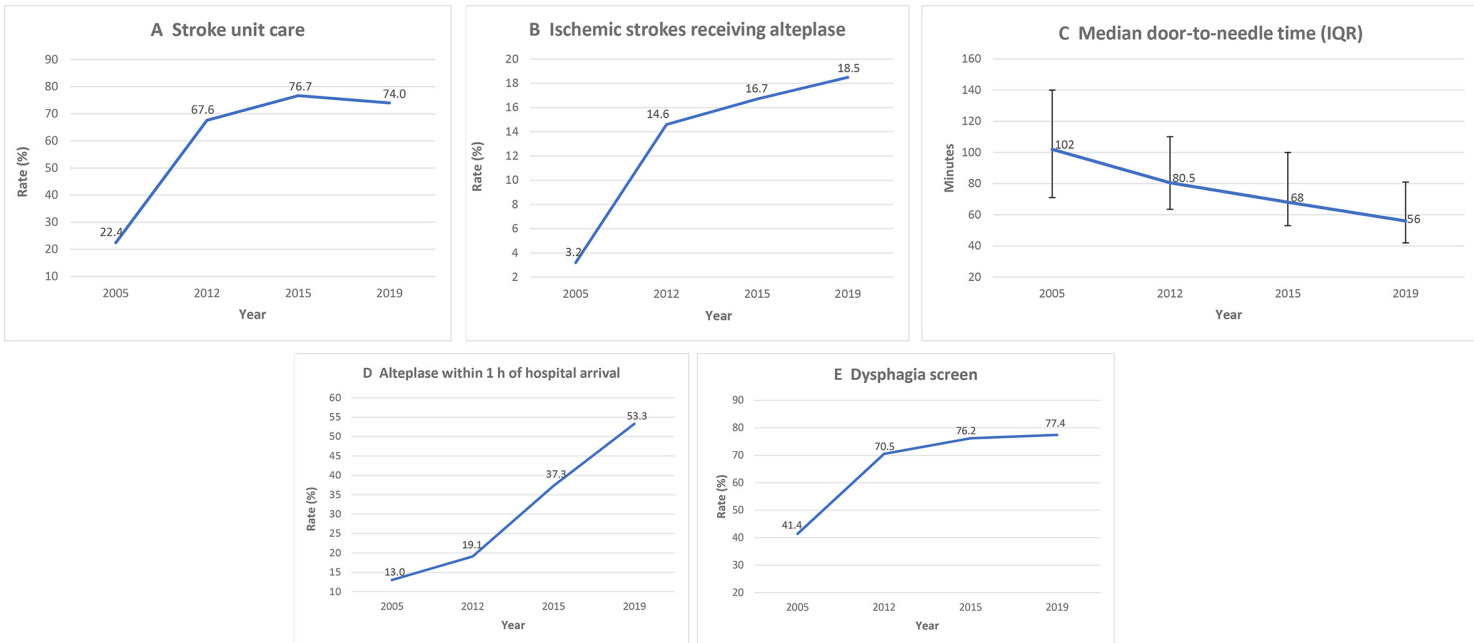

Figure 1 Charts showing the trajectory of improvement from preintervention levels in 2005 for: (A) Proportion of patients receiving stroke unit care; (B) Proportion of ischaemic patients who had a stroke receiving treatment with intravenous alteplase; (C) Median door-to-needle times for patients treated with intravenous alteplase; (D) The proportion of patients receiving alteplase within 1 hour of hospital arrival; and (E) Proportion of patients receiving a dysphagia screen in the emergency department.

although in one DHA it was not possible to establish a six-bed unit, geography dictated the need for a unit in that area. In all facilities, patients who had a stroke were clustered on an existing unit. At the QEII, this was an acute neurology unit; elsewhere, patients who had a stroke were clustered on general medical units.

In a consultation process that involved CVHNS and the DHAs, the results of the 2004-2005 audit were used to conduct a gap analysis that described the disparities between best practice recommendations and actual care delivery in each DHA. The DHAs were then required to submit proposals for improvements that would establish a DSP and close the gaps in the delivery of their local stroke services.
Gaps varied between DHAs and local teams decided their priority initiatives to meet best practices; consequently, the starting points for improvement were different in each DHA, and there were multiple improvement initiatives with different aims running concurrently province wide. Early adopters were encouraged to share their experiences with other DHAs at CVHNS-led provincial forums.

To facilitate change, CVHNS provided direction and support through the network of DSPs, as well as fiscal supervision (to ensure that funds were spent as planned), and various educational opportunities. CVHNS used a combination of strategies ${ }^{17}$ recognising that staggered implementation required flexibility and

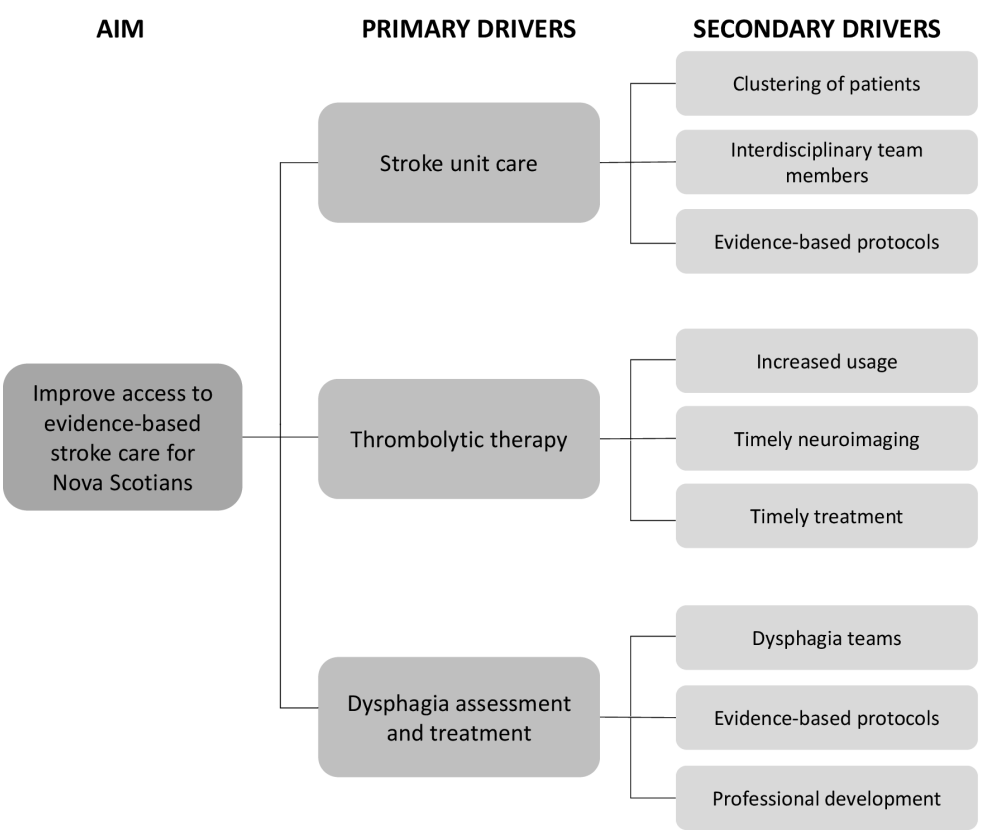

CHANGE IDEAS

Cluster patients on one medical unit Protocols, target times for transfers to stroke unit Enhance team to meet standards for size of unit Evidence-based provincial and local education Standardized order sets, policies, procedures Local data collection \& provincial monitoring Learning \& sharing forum

Evidence-based provincial and local education Code Stroke protocols Pre-hospital notification \& identification Patient direct to CT scanner on arrival. Patient to CT scanner on ambulance stretcher Next-in-scanner (no wait) protocol Radiologist phones physician with scan results Standardized eligibility checklist and order set Ease of access to alteplase, checklist and order set Webinar series to monitor \& share local outcomes

Train-the-trainer sessions with team members Standardized tool for swallow screening Protocols for nursing dysphagia assessment Virtual training packages Support for attendance at national workshops

Figure 2 Driver diagram illustrating approach to improving stroke care in Nova Scotia. 


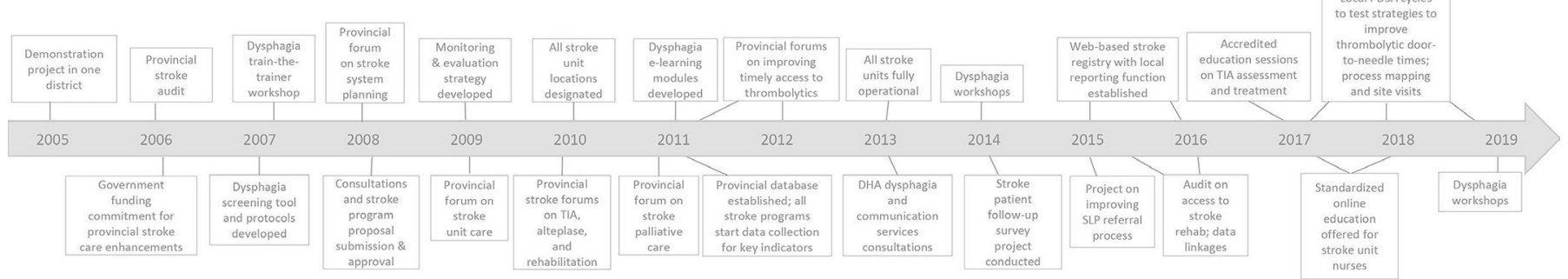

Figure 3 Implementation activities, 2005-2019. DHA, district health authority; PDSA, plan-do-study-act; SLP, speechlanguage pathology; TIA, transient ischaemic attack.

non-linear approaches. ${ }^{18}$ Key implementation processes are summarised in box 1, subdivided by CVHNS activities, and actions by the DHAs and DSPs. The progression of improvement efforts over time is displayed in figure 3 .

\section{Stroke unit care}

The first priority of reorganisation was to facilitate coordinated multidisciplinary stroke unit care because this had the greatest potential to benefit the largest number of patients. ${ }^{81920}$ A stroke unit was defined as a geographically defined ward area, where coordinated care is provided by a specialised, experienced stroke team. Stroke team capacity in each hospital prior to reorganisation was estimated from the audit data. Staffing targets were based on data from the QEII, ${ }^{16}$ and the levels achieved in Ontario hospitals following implementation of the Ontario Stroke

\section{Box 1 Key implementation processes}

\section{Cardiovascular Health Nova Scotia activities}

- Development of a network to facilitate ongoing collaboration and structured information exchange

- Creation of the stroke programme coordinator and stroke programme lead physician roles to foster local leadership, collaboration and knowledge brokering

- Hosting of provincial forums for local teams for the purposes of learning, sharing and planning

- Creation of a provincial stroke registry for standardised data collection

- Identification of care gaps through monitoring

- Provision of professional education opportunities to hone healthcare improvement skills, uptake of best practice guidelines and use of decision support tools

- Provision of implementation milestone targets

\section{Actions by local health system partners}

- Creation of a steering or advisory committee with representatives from all settings and sites

- Tracking of, and reporting on, non-portable funding

> Development of process maps, and assessment of local strengths and opportunities for improvement

- Provision of feedback on performance for front-line staff

- Implementation of process changes, including but not confined to:

- Ambulance acute stroke bypass protocols

- Rapid access to CT scanning and image interpretation

- Thrombolysis protocols

- Rapid access to stroke prevention services for patients with transient ischaemic attack
Strategy. ${ }^{21}$ Additional information was sought from published studies, guideline statements and the Canadian Stroke Network; for example, adverse outcomes had been shown to be more frequent in low-volume facilities $(<50$ strokes/year) than in high-volume facilities $(>100$ strokes/year). ${ }^{22}$

The core staffing complement per 10-bed stroke unit was estimated as: 0.8 full-time equivalent (FTE) dietitian; 14 FTE nurses; 1.0 specialty nurse practitioner; 1.0 FTE occupational therapist, physiotherapist and speechlanguage pathologist; and 0.5 FTE social worker. Staffing levels (nursing excepted) on the district stroke teams were enhanced according to this formula using the new funding from the department of health. Additional disciplines (eg, pharmacy, recreation therapy), funded separately, were included in some stroke teams. The structure and function of stroke unit care varied between facilities, but all provided care according to evidence-based protocols, and had regular team meetings and access to ongoing education.

The implementation process for stroke unit care involved a 1-day in-person conference for education, planning and information sharing. Additional provincial forums focused on systems reorganisation, quality indicators, thrombolytic therapy, palliative care, rehabilitation treatment, and the management of TIA and nondisabling stroke (figure 3). Forums were structured to provide an avenue for learning, sharing and planning for local improvements.

\section{Thrombolytic therapy}

Policies, procedures and protocols were developed and implemented to facilitate timely treatment of acute ischaemic stroke with intravenous alteplase. In collaboration with emergency health services, paramedics were trained to use the Cincinnati Prehospital Stroke Scale ${ }^{23}$ to screen patients for possible stroke; as well, bypass protocols were established to allow paramedics transporting patients with suspected acute stroke within the time window for thrombolysis to proceed directly to the nearest designated acute stroke hospital, rather than taking the patient to a closer facility not equipped with a CT scanner. Day-to-day clinical support for physicians making diagnostic and therapeutic decisions about acute stroke outside the QEII was provided by members of the division of neurology as part of their routine $24 / 7$ on-call 
work. Teleconsultation was at the discretion of the physician in the remote hospital, and typically involved a telephone conversation and review of the CT images on the provincial computerised picture archiving and communication system.

\section{Dysphagia screening and assessment}

CVHNS, in partnership with the Heart and Stroke Foundation of Nova Scotia and the Nova Scotia Hearing and Speech Centres (NSHSC), developed two tools for dysphagia screening and assessment: a Stroke Swallow Screen and a Nursing Dysphagia Assessment Protocol. In 2007, CVHNS and NSHSC offered a 3-day workshop for dysphagia teams (speech-language pathologists, dietitians and nurses) from all DHAs. The workshop used a 'trainthe-trainer' model, with the goals of increasing participants' knowledge and skills in dysphagia screening and assessment, implementing best practices, and providing opportunities for an interdisciplinary team approach. The participants returned to their local stroke programmes to conduct training sessions using the knowledge and skills gained from the workshop. E-learning modules were developed in 2011 for both the stroke swallow screening tool and the nursing dysphagia assessment protocol to support self-directed learning at the local level. CVHNS and NSHSC offered additional in-person dysphagia team workshops in 2014 and 2019.

\section{RESULTS}

The quantitative results are shown in table 1 and figure 1 .

The crude annual hospitalisation rate for stroke and TIA increased over time. The median age of the patients decreased from 77 to 74 , mainly because of an increase in the hospitalisation rate among individuals under age 50 , and a decrease in the rate for individuals aged 80 or more. Sex distribution remained unchanged.

The impact of a steady increase in the use of brain imaging was reflected in improved diagnostic precision, that is, a reduction in the proportion of patients who were classified as having a stroke of unknown type. The proportion of patients receiving dysphagia screening and multidisciplinary stroke unit care increased rapidly and substantially but then levelled off (the apparent drop in the proportion of patients assessed by a speech-language pathologist is an artefact of a change in referral patterns and data collection processes in 2018). Contrastingly, the metrics for thrombolytic therapy showed continued steady improvement over the 14-year interval, without an increase in the proportion of patients experiencing the complication of intracranial haemorrhage.

More patients were transferred from acute care to a specialised in-patient rehabilitation unit, and fewer were discharged to residential or long-term care. However, there was no observed change in unadjusted 30-day in-hospital mortality or the proportion of patients who were functionally independent at the time of discharge from hospital (though documentation of modified Rankin
Box 2 Stakeholder feedback on lessons learnt

Key success factors
Experience of the preimplementation demonstration project in rural
Nova Scotia
Leadership, support and guidance at provincial and local levels
- Local: stroke physician champion and stroke coordinator roles
- Provincial: Cardiovascular Health Nova Scotia
Stroke teams that were collaborative, creative, committed and
flexible
Active engagement (conversations, planning, decision-making), re-
lationship building, ownership and support from/across all levels of
the healthcare system
Culture of participation and contribution-share, spread, improve
and share again
Staff education in a variety of formats-locally, provincially and
nationally
Supporting policies and protocols
Frequent networking opportunities-provincial forums, and among
stroke programme coordinators
Patient-centred focus
Celebrations of success
- Timely monitoring of key indicators providing opportunities for
improvement
Key challenges
- Competing turied health professionals fulfilling multiple roles where resources
were limited
Bed flow
Physician buy-in and agreement with guidelines and protocols
Resing taff

scores was missing in about $8 \%$ of patients in 2012-2019). The changes in care processes did not influence median length of stay in hospital.

The qualitative stakeholder feedback data are summarised thematically in box 2 . The network was a critical success factor while competing priorities in the healthcare system were the main challenge to implementing change.

\section{Strengths, lessons and limitations}

The main strength of this work is that it was a long-term, real-world implementation project conducted across an entire provincial healthcare jurisdiction, not a limited research project conducted in an academic healthcare centre. In the course of our implementation efforts, we learnt that moving knowledge into action within healthcare systems is complex ${ }^{24}$ and deliberate effort is required in order to 'make things happen' rather than 'let things happen'. ${ }^{25}$ Specifically, we attributed the substantial improvements in process outcomes to the impact of the stroke physician and stroke coordinator roles in the domains of knowledge brokering, knowledge transfer teams and network facilitation, which were demonstrated effective in improving stroke care in Australia ${ }^{26} 27$ and other parts of Canada. ${ }^{28-31}$ At the same time, we were 
challenged by the constraints and limitations of our healthcare system (box 2, key challenges).

The initiative to coalesce the previously fragmented and disorganised management of patients who had a stroke in 33 hospitals province wide into coordinated multidisciplinary stroke unit care provided within a network of seven stroke programmes worked well and quickly; yet, the proportion of patients admitted to a stroke unit levelled off at around 75\%, well below the 95\% figure reported in the 2018 UK Sentinel Stroke National Audit Programme. ${ }^{32}$ The CVHNS Stroke Registry did not capture the reasons for patients not receiving stroke unit care, though we suspect that bed capacity issues were mainly responsible.

The urgency and metrics around thrombolytic therapy lend themselves well to quality improvement work. Our multidimensional intervention did not include videoteleconferencing $^{33}$ with the on-call neurologist at the QEII ('telestroke, ${ }^{34}$ ) yet increased the proportion of patients receiving alteplase from $3.2 \%$ in 2005 to $18.5 \%$ in 2019 (figure 1B), which is close to the estimated maximum of $24 \% .{ }^{35}$ Our real-world evidence complements the findings from a recent systematic review and meta-analysis of interventions to increase stroke thrombolysis, ${ }^{36}$ and compares favourably with the experience of the well-known TeleMedical Project for integrative Stroke Care project in rural Bavaria where the rates of alteplase administration increased from $0.4 \%$ in the year before telestroke implementation (2002) to $15.5 \%$ in the intervention's 10th year (2012) ${ }^{37}$ Telestroke has been criticised because of concerns about workloads among the relatively small number of stroke neurologists providing the service. ${ }^{38}$ Our experience in Nova Scotia suggests that videoconferencing with a neurologist for thrombolysis decisions is not required and that building capacity locally may be a more acceptable and sustainable way of enhancing acute stroke treatment.

Our interventions also improved the efficiency of thrombolytic therapy (figure $1 \mathrm{C}, \mathrm{D}$ ). While our results compare favourably with other recent population-level studies reporting $32 \%-61 \%$ of patients treated within $60 \mathrm{~min},{ }^{39-43}$ we remain uncertain whether it will be possible to uniformly and sustainably attain the current guideline target of a median door-to-needle time of $30 \mathrm{~min}^{44}{ }^{45}$ at thrombolysing hospitals in Nova Scotia.

Dysphagia screening utilisation seemed to plateau (figure 1E) just below the recommended target of at least $80 \% .^{5}$ Contributing factors may have been staff turnover, and delays in the approval and distribution of a revised provincial screening tool. For a variety of reasons, we think that $100 \%$ compliance seems unattainable.

We were reminded that, for meaningful interpretation, comparisons of mortality and functional status require adjustment for case mix. ${ }^{46}$ We did not demonstrate any impact on unadjusted 30-day in-hospital mortality (table 1); however, CIHI (using a case definition different from ours) reported $^{48}$ a decline in 30-day stroke in-hospital mortality in Nova Scotia from $25.1 \%$ in 2005 to $10.0 \%$ in 2019, when adjusted for age, sex, and comorbidity quantified using the Charlson Index Score (not captured by the CVHNS Stroke Registry). As well, a Canadian study ${ }^{49}$ that used CIHI data from 2004 to 2013 showed a greater reduction in 30-day in-hospital mortality in provinces with integrated systems of stroke care (Nova Scotia included) than in provinces without such systems.

Other limitations are that our experience is generalisable only to similar single-payer healthcare systems. We did not include the view from the patients' perspective ${ }^{47}$ or capture any information about patients who had a stroke but were not admitted to hospital. Nor did we capture stroke severity, poststroke complications, quality of life or any details about rehabilitation treatment, community reintegration and longer-term functional outcome.

\section{CONCLUSION}

Stroke care reorganisation generated substantial improvements in process outcomes across the entire Canadian provincial healthcare jurisdiction of Nova Scotia between 2005 and 2019. Patients who had a stroke gained increased access to, and timelier administration of, best practice investigations and interventions, including brain imaging, thrombolytic therapy and specialised in-patient rehabilitation treatment, without an increase in length of stay in acute care. A combination of interventions and healthcare improvement strategies effected continuous change in all designated stroke hospitals. Maintenance of a functioning province-wide network and the provision of objective feedback using data from the CVHNS Stroke Registry helped sustain performance, and adherence to defined best practice recommendations. Continuous monitoring enabled flexibility, in terms of report generation, and collecting information without consent minimised ascertainment bias. $^{50}$

The results of this study have prompted us to reconsider different ways of blending the use of CIHI data with combined continuous monitoring and periodic targeted audit by CVHNS to measure the impact of future quality improvement efforts. ${ }^{47}$ Ad Aditionally, our accomplishments provide a foundation for further efforts targeted at rehabilitation and community reintegration, and the equitable provision of endovascular thrombectomy. ${ }^{52}$ We think that the network we created has the potential to expand our capacity for clinical and health services research. As well, the learning from our experience in bringing about improvements across the broad continuum of stroke care from the prehospital phase through acute care and rehabilitation may find application in the management of other conditions. Moving forward, the main challenge will be to preserve and enhance the commitment and cohesion at all levels of the healthcare system in order to match our resources against the complexity and magnitude of stroke.

The improvement work described here was conducted within an envelope of funding from the provincial government that was used mainly to enhance stroke team staffing. Although lack of resources is often perceived as a barrier to 
system change ${ }^{53}$ we demonstrated that much can be accomplished through reorganisation of existing services. We did not conduct a cost analysis, but other studies have demonstrated the cost-effectiveness of thrombolytic therapy, ${ }^{54}$ and it has been estimated that optimising comprehensive stroke care in Canada could avoid costs of $\$ 682$ million annually. ${ }^{55}$

Acknowledgements For the 2005 audit, we acknowledge the support from the Institute for Clinical Evaluative Sciences and the former Canadian Stroke Network, and thank abstractors Susan Atkinson, Mamie Curley, Kelly Goudey, Lena MacDonald, Kim MacKenzie, Valerie Mackie, Glenda 0'Reilly and Cheryl Smith. The improvements highlighted here are the result of the combined efforts of many current and former Nova Scotia Health Authority staff, particularly: Cardiovascular Health Nova Scotia Stroke consultants: Corinne Corning, Wendy Simpkin, Allison Stevens, Schelene Swinemar, and Katie White. Stroke physician champions: Mohammad Fahim, Mary Gorman, Mandat Maharaj, David Marsters, Graham Miles, Michael Mindrum, Brian Moses, Henry Onyegbule, Heather Robertson, Laith Shimon and Kris Srivatsa. Stroke coordinators: Lesley Austin-Smith, Meaghan Bushell, Sandy Cantwell-Kerr, Andrea Cottrell, Christina Darrigan-Skinner, Kelly Goudey, Neil Hewitt-Barney, Judith Hussey, Tracy MacGillivary, Michelle MacGrath, Deb Mander, Deborah Merrick, Melanie Mooney, Linda Sears-Doucet, Wendy Simpkin, Elizabeth Stainwright, Nicholas Swift, Schelene Swinemar, Tina Vardy, Jennifer White and Heather Wolfe. QEIl stroke physicians: Laine Green, Gord Gubitz, Brenda Joyce, Anita Mountain, Amra Saric and Sean Taylor. QEIl stroke nurse practitioners: Krista Biggs, Christine Bryden, Mary-Elizabeth Cooper, Sonia Kettle, Karen Legg, Michelle MacKay, Angela Meagher, Shannon Nearing and Lindsay Tennyson.

Contributors SJP conceived the study and wrote the manuscript. AS was stroke consultant for Cardiovascular Health Nova Scotia (CVHNS), created the graphics, assisted with analyses and critiqued the manuscript. $\mathrm{HC}$ provided biostatistical expertise and ran the data analyses. WS collected data for the provincial audit, assisted with analyses and critiqued the manuscript. JP provided epidemiological expertise and critiqued the manuscript. NG was manager of CVHNS, led the interventions and critiqued the manuscript.

Funding The authors have not declared a specific grant for this research from any funding agency in the public, commercial or not-for-profit sectors.

Competing interests None declared.

Patient and public involvement Patients and/or the public were not involved in the design, or conduct, or reporting, or dissemination plans of this research.

Patient consent for publication Not required.

Ethics approval This study was deemed by the Department of Health and Wellness of the government of Nova Scotia, Canada not to require consent from the participants because the work focused on improving the quality of healthcare delivery in the provincial jurisdiction of Nova Scotia.

Provenance and peer review Not commissioned; externally peer reviewed.

Data availability statement Data may be obtained from a third party and are not publicly available.

Open access This is an open access article distributed in accordance with the Creative Commons Attribution Non Commercial (CC BY-NC 4.0) license, which permits others to distribute, remix, adapt, build upon this work non-commercially, and license their derivative works on different terms, provided the original work is properly cited, appropriate credit is given, any changes made indicated, and the use is non-commercial. See: http://creativecommons.org/licenses/by-nc/4.0/.

ORCID iD

Stephen James Phillips http://orcid.org/0000-0002-7880-9358

\section{REFERENCES}

1 Langhorne P, Williams BO, Gilchrist W, et al. Do stroke units save lives? Lancet 1993;342:395-8.

2 National Institute of Neurological Disorders and Stroke rt-PA Stroke Study Group. Tissue plasminogen activator for acute ischemic stroke. N Engl J Med 1995;333:1581-8.

3 Black D, Lewis M, Monaghan B, et al. System change in healthcare: the Ontario stroke strategy. Hosp Q 2003;6:44-7.

4 Lindsay P, Bayley M, McDonald A, et al. Toward a more effective approach to stroke: Canadian best practice recommendations for stroke care. Can Med Assoc J 2008;178:1418-25.
5 Canadian stroke best practices. Available: www.strokebestpractices.

6 Biller J, Love BB. Nihilism and stroke therapy. Stroke 1991:22:1105-7.

7 Wilson E, Taylor G, Phillips S, et al. Creating a Canadian stroke system. CMAJ 2001;164:1853-5.

8 Langhorne P, Ramachandra S, Stroke Unit Trialists' Collaboration. Organised inpatient (stroke unit) care for stroke: network metaanalysis. Cochrane Database Syst Rev 2020;4:CD000197.

9 Langhorne P, Pollock A, Stroke Unit Trialists' Collaboration. What are the components of effective stroke unit care? Age Ageing 2002;31:365-71.

10 Kamal N, Smith EE, Jeerakathil T, et al. Thrombolysis: Improving door-to-needle times for ischemic stroke treatment - A narrative review. Int J Stroke 2018;13:268-76.

11 Canadian Stroke Network. The quality of stroke care in Canada, 2011. Available: http://www.canadianstroke.ca/sites/default/files/ resources/QoSC-EN.pdf

12 Lindsay P, Furie KL, Davis SM, et al. World stroke organization global stroke services guidelines and action plan. Int J Stroke 2014;9 Suppl A100:4-13.

13 Kapral MK, Laupacis A, Phillips SJ, et al. Stroke care delivery in institutions participating in the registry of the Canadian stroke network. Stroke 2004;35:1756-62.

14 Canadian Institute for health information discharge Abstract database. Available: cihi.ca/en/discharge-abstract-databasemetadata-dad

15 van Swieten JC, Koudstaal PJ, Visser MC, et al. Interobserver agreement for the assessment of handicap in stroke patients. Stroke 1988;19:604-7.

16 Phillips SJ, Eskes GA, Gubitz GJ, et al. Description and evaluation of an acute stroke unit. CMAJ 2002;167:655-60.

17 Eccles M, Grimshaw J, Walker A, et al. Changing the behavior of healthcare professionals: the use of theory in promoting the uptake of research findings. J Clin Epidemiol 2005;58:107-12.

18 Handley MA, Gorukanti A, Cattamanchi A. Strategies for implementing implementation science: a methodological overview. Emerg Med J 2016;33:660-4.

19 Hankey GJ, Warlow CP. Treatment and secondary prevention of stroke: evidence, costs, and effects on individuals and populations. Lancet 1999:354:1457-63.

20 Gilligan AK, Thrift AG, Sturm JW, et al. Stroke units, tissue plasminogen activator, aspirin and neuroprotection: which stroke intervention could provide the greatest community benefit? Cerebrovasc Dis 2005;20:239-44.

21 Integrated stroke care in Ontario: stroke evaluation report. (2007). heart and stroke Foundation of Ontario. Available: http://www.cana dianstrokestrategy.ca/

22 Saposnik G, Baibergenova A, O'Donnell M, et al. Hospital volume and stroke outcome: does it matter? Neurology 2007;69:1142-51.

23 Kothari RU, Pancioli A, Liu T, et al. Cincinnati prehospital stroke scale: reproducibility and validity. Ann Emerg Med 1999;33:373-8.

24 Graham ID, Logan J, Harrison MB, et al. Lost in knowledge translation: time for a MAP? J Contin Educ Health Prof 2006;26:13-24

25 Greenhalgh T, Robert G, Macfarlane F, et al. Diffusion of innovations in service organizations: systematic review and recommendations. Milbank Q 2004;82:581-629.

26 Cadilhac DA, Purvis T, Kilkenny MF, et al. Evaluation of rural stroke services: does implementation of coordinators and pathways improve care in rural hospitals? Stroke 2013;44:2848-53.

27 Purvis T, Moss K, Denisenko S, et al. Implementation of evidencebased stroke care: enablers, barriers, and the role of facilitators. $J$ Multidiscip Healthc 2014;7:389-400.

28 Cameron JI, Rappolt S, Lewis M, et al. Development and implementation of the Ontario stroke system: the use of evidence. Int $J$ Integr Care 2007;7:e30.

29 Dobbins $M$, Robeson P, Ciliska D, et al. A description of a knowledge broker role implemented as part of a randomized controlled trial evaluating three knowledge translation strategies. Implement Sci 2009;4:23.

30 Warner G, Lyons R, Parker V, et al. Advancing coordinated care in four provincial healthcare systems: evaluating a knowledgeexchange intervention. Healthc Policy 2011;7:80-94.

31 Singh M, Hynie M, Rivera T, et al. An evaluation study of the implementation of stroke best practice guidelines using a knowledge transfer team approach. Can J Neurosci Nurs 2015;37:24-33.

32 Sentinel stroke national audit programme (SSNAP). clinical audit April 2013 - March 2018. annual public report. Available: https://www. hqip.org.uk/resource/sentinel-stroke-national-audit-programmeannual-report-2019 
33 Blacquiere D, Lindsay MP, Foley N, et al. Canadian stroke best practice recommendations: TeleStroke best practice guidelines update 2017. Int J Stroke 2017;12:886-95.

34 Levine SR, Gorman M. "Telestroke" : the application of telemedicine for stroke. Stroke 1999;30:464-9.

35 Boode B, Welzen V, Franke C, et al. Estimating the number of stroke patients eligible for thrombolytic treatment if delay could be avoided. Cerebrovasc Dis 2007;23:294-8.

36 McDermott M, Skolarus LE, Burke JF. A systematic review and meta-analysis of interventions to increase stroke thrombolysis. BMC Neurol 2019;19:86.

37 Müller-Barna P, Hubert GJ, Boy S, et al. Telestroke units serving as a model of care in rural areas: 10-year experience of the telemedical project for integrative stroke care. Stroke 2014;45:2739-44.

38 French B, Day E, Watkins $C$, et al. The challenges of implementing a TeleStroke network: a systematic review and case study. BMC Med Inform Decis Mak 2013;13:125 http://www.biomedcentral.com/1472 $6947 / 13 / 125$

39 Stroke Foundation. National stroke audit - acute services report 2019. Melbourne, Australia. Available: https://informme.org.au/ stroke-data/media

40 Kamal N, Sheng S, Xian Y, et al. Delays in door-to-needle times and their impact on treatment time and outcomes in get with the Guidelines-Stroke. Stroke 2017;48:946-54.

41 Liu Q, Ranta AA, Abernethy G, et al. Trends in New Zealand stroke thrombolysis treatment rates. N Z Med J 2017;130:50-6.

42 Hsieh F-I, Jeng J-S, Chern C-M, et al. Quality improvement in acute ischemic stroke care in Taiwan: the breakthrough collaborative in stroke. PLoS One 2016;11:e0160426.

43 McVerry F, Hunter A, Dynan K, et al. Country-wide analysis of systemic factors associated with acute ischemic stroke door to needle time. Front Neurol 2019;10:676.

44 Kamal N, Benavente O, Boyle K, et al. Good is not good enough: the benchmark stroke door-to-needle time should be 30 minutes. Can $\mathrm{J}$ Neurol Sci 2014;41:694-6.
45 Boulanger JM, Lindsay MP, Gubitz G, et al. Canadian stroke best practice recommendations for acute stroke management: prehospital, emergency department, and acute inpatient stroke care, 6th edition, update 2018. Int J Stroke 2018;13:949-84.

46 Davenport RJ, Dennis MS, Warlow CP. Effect of correcting outcome data for case mix: an example from stroke medicine. BMJ 1996;312:1503-5.

47 Walsh K, Gompertz PH, Rudd AG. Stroke care: how do we measure quality? Postgrad Med J 2002;78:322-6.

48 Canadian Institute for Health Information. Health indicators interactive tool. Available: http://yourhealthsystem.cihi.ca/epub/? language $=$ en

49 Ganesh A, Lindsay P, Fang J, et al. Integrated systems of stroke care and reduction in 30-day mortality: a retrospective analysis. Neurology 2016;86:898-904.

50 Tu JV, Willison DJ, Silver FL, et al. Impracticability of informed consent in the registry of the Canadian stroke network. N Engl J Med 2004;350:1414-21.

51 Palmer WL, Bottle A, Davie C, et al. Meeting the ambition of measuring the quality of hospitals' stroke care using routinely collected administrative data: a feasibility study. Int J Qual Health C 2013;25:429-36.

52 Hu SX, Virani K, Shankar JJS, et al. Case-Control study of endovascular thrombectomy in a Canadian stroke center. Can J Neurol Sci 2020;47:44-9.

53 Gache K, Leleu H, Nitenberg G, et al. Main barriers to effective implementation of stroke care pathways in France: a qualitative study. BMC Health Serv Res 2014;14:95 http://www.biomedcentral. com/1472-6963/14/95

54 Joo H, Wang G, George MG. A literature review of costeffectiveness of intravenous recombinant tissue plasminogen activator for treating acute ischemic stroke. Stroke Vasc Neurol 2017;2:73-83.

55 Krueger $\mathrm{H}$, Lindsay $\mathrm{P}$, Cote $\mathrm{R}$, et al. Cost avoidance associated with optimal stroke care in Canada. Stroke 2012;43:2198-206. 